\title{
Effets du scarifiage sur les propriétés du sol, la croissance des semis et la compétition : revue des connaissances actuelles et perspectives de recherches au Québec
}

\author{
M Prévost
}

\begin{abstract}
Ministère des forêts du Québec, service de la recherche appliquée, 2700 rue Einstein, Sainte-Foy, Québec, G1P 3 W8 Canada
\end{abstract}

(Reçu le 22 avril 1991; accepté le 23 janvier 1992)

\begin{abstract}
Résumé - La remise en production des aires de coupe est maintenant un élément important de la stratégie sylvicole du Québec. Elle est, d'ailleurs, inscrite dans la loi sur les forêts qui est l'élément législatif majeur de la politique forestière québécoise. La préparation de terrain après coupe, préalablement au reboisement ou plus rarement avant ensemencement, est souvent utilisée pour faciliter l'établissement des semis. Au Québec, le scarifiage, qui mélange le sol organique au sol minéral ou enlève en tout ou en partie l'horizon organique, est, de loin, le traitement le plus fréquemment appliqué sur les sites permettant le passage de la machinerie. Cet article fait la synthèse des connaissances actuelles concernant les effets du scarifiage sur la réaction des semis et sur les paramètres environnementaux qui influencent cette dernière. Les effets sur les propriétés du sol (densité apparente, régimes hydrique, thermique et nutritif), sur l'établissement et la croissance des semis et sur le développement de la végétation compétitrice sont examinés. Malgré une utilisation toujours accrue du scarifiage lors de la préparation de terrain au Québec, encore très peu de recherches ont décrit les impacts de ce traitement, et les besoins de connaissances spécifiques à nos conditions écologiques demeurent.
\end{abstract}

préparation de terrain / scarifiage / propriété du sol / établissement des semis / régénération forestière

Summary - Effects of scarification on soil properties, seedling growth and competition: review of current knowledge and research perspectives in Quebec. Regeneration of harvested areas is now an important element of the silvicultural strategy of Quebec. Moreover, it is written into the law on the forests which is the major legislative element in Quebec forest policy. Site preparation after cutting, either prior to plantation or more rarely before seeding, is often carried out to facilitate seedling establishment. In Quebec, scarification, which mixes mineral and organic soil or removes a certain depth of organic matter, is by far the most frequently applied treatment on trafficable sites. This paper reviews current knowledge about effects of scarification on both seedling response and on environmental parameters influencing this response. The effects on soil properties (bulk density, and moisture, thermal and nutrient regimes), on seedling establishment and growth, and on development of competing vegetation are examined. In spite of the increasing use of scarification as a site preparation technique in Quebec, little research has been undertaken on the impact of this treatment, and more specific knowledge of our particular ecological conditions is required. 


\section{INTRODUCTION}

La préparation de terrain après coupe est devenue une pratique courante en foresterie pour palier les échecs de la régénération naturelle. Elle vise l'enlèvement des débris de coupe et de la végétation compétitrice pour créer un environnement favorable à l'établissement et à la croissance des semis (Orlander et al, 1990). Le plus souvent, elle vient faciliter l'établissement de plantations et quelquefois, sert à promouvoir la venue de régénération naturelle à partir de semenciers laissés sur pied. Trois types de traitements sont généralement utilisés, séparément ou de façon combinée pour la préparation de terrain : le brûlage contrôlé, les phytocides et les méthodes mécaniques. Le choix du traitement dépend avant tout des conditions du site (déchets de coupe, végétation indésirable, sol, pente). II devrait idéalement être dicté par la nature et l'intensité des modifications désirées (Fryk, 1986), mais demeure limité par la disponibilité de l'équipement et le manque de connaissances.

En général, le brûlage n'est utilisé que lorsque les débris de coupe et la végétation ind sirable sont abondants. Au Canada, cette méthode est utilisée avec succès en Ontario où les coupes avec ébranchage sur place laissent une abondance de rémanents. Elle est largement employée en Colombie Britannique où la quantité et la taille des débris ainsi que les pentes difficiles limitent l'accès de la machinerie. En raison des dangers de perte de contrôle du feu, l'application du brûlage requiert à tout le moins des conditions météorologiques favorables (Hedin, 1986). D'autre part, l'utilisation de phytocides est peu répandue au Canada pour la préparation de terrain, puisqu'une opération de scarifiage s'avère de toute façon nécessaire pour enlever les résidus après traitement (Smith, 1984). Alors que nous sommes de plus en plus conscients de la fragilité de la biosphère, l'emploi de phytocides soulève l'interrogation quant aux impacts environnementaux et se fait surtout à titre de complément aux autres méthodes. Le scarifiage est, par ailleurs, une technique peu polluante qui favorise l'incorporation de la matière organique au sol minéral tout en permettant un contrôle de la végétation compétitrice. Au Canada, la mécanisation des opérations forestières et la nécessité d'adapter les travaux de préparation de terrain à la technologie moderne ont fait que le scarifiage était utilisé sur plus des $2 / 3$ des superficies traitées au début des années 1980 (Sutherland, 1987).

Au Québec, plus de 50000 ha ont été scarifiés sur les terres publiques en 1986 (Fortin et Im, 1987). Pour la grande majorité des travaux, des appareils scandinaves ont été utilisés et les perspectives laissaient entrevoir leur utilisation accrue pour l'avenir. Or, il importe de connaître les possibilités d'appliquer au Québec cette technique développée sous des conditions différentes. Cet article fait la revue des connaissances actuelles en matière de scarifiage en faisant ressortir ses effets sur les propriétés du sol (densité apparente, disponibilité en eau, régime thermique, fertilité), ainsi que sur la réaction des semis et de la végétation compétitrice. II vise finalement à faire ressortir les axes de recherches pour une application de ce type de traitement dans le contexte québécois. À ce jour, encore peu de travaux de recherches ont mesuré la réponse biologique des semis à la suite d'un scarifiage sur des sites canadiens. Des études comparatives ont été réalisées principalement en Ontario et en Colombie Britannique. Des recherches spécifiques sont nécessaires pour déterminer les effets réels de ce traitement du sol sous les conditions québécoises. 


\section{IMPORTANCE DES PROPRIÉTÉS DU SOL POUR LA CROISSANCE DES PLANTES}

Le sol est un milieu poreux déformable qui sert de support physique pour les plantes. II est aussi à la fois un réservoir et un conducteur pour les éléments nutritits, l'eau et l'air (Vomocil et Flocker, 1961). Les propriétés du sol qui influencent l'enracinement sont donc d'une grande importance (Lévy, 1968), puisqu'elles déterminent indirectement l'efficacité du système racinaire pour l'ancrage et le prélèvement d'eau et de nutriments. Ces propriétés du sol sont des caractéristiques mesurables susceptibles d'être modifiées par le scarifiage.

\section{Disponibilité de l'eau}

La disponibilité de l'eau est un facteur susceptible d'influencer la plupart des processus physiologiques et biochimiques des plantes. L'eau est un réactif dans la photosynthèse, elle est le principal constituant du protoplasme et est essentielle au maintien de la turgescence des cellules (Kramer, 1962). Étant à la base de la production primaire, le processus de diffusion du dioxyde de carbone par les stomates s'accompagne de pertes inévitables d'eau par transpiration (Rutter, 1975). Lorsque les réserves en eau du sol s'épuisent, les pertes ne sont plus compensées, ce qui provoque un stress hydrique à l'intérieur de la plante. La réaction de défense est la fermeture des stomates, inévitablement accompagnée par une baisse de la photosynthèse. Le stress hydrique affecte également des processus tels le métabolisme de l'azote, l'absorption des sels, la translocation et l'accroissement des cellules (Kramer, 1962). En outre, l'eau est néc:essaire pour le transport des nutriments du sol vers les racines (Becker et Lévy, 1983) et un dessèchement du sol réduit la biomasse racinaire (Becker, 1977).

L'eau est retenue dans la matrice de sol par adsorption à la surface des particules et par capillarité dans les pores (tension matricielle). La force de rétention et le volume disponible dépendent de la distribution et de la dimension des pores. De façon générale, on estime que l'eau disponible pour la plante est le volume contenu entre les limites approximatives de 30 et $1500 \mathrm{kPa}$ de tension (fig 1). En deçà d'environ $30 \mathrm{kPa}$ (capacité au champ), l'eau contenue dans les macropores (> 10 à 50 $\mu \mathrm{m})$ se draine rapidement par gravité (12 j). Au-delà de $1500 \mathrm{kPa}$ (point de flétrissement), elle est très fortement retenue dans les micropores $(<0,2 \mu \mathrm{m})$ et ne peut être puisée en quantité suffisante pour maintenir la turgescence des feuilles. Le volume d'eau disponible est minimal pour le sable grossier et maximal pour les sols à texture moyenne.

\section{Régime thermique}

Le régime thermique du sol influence directement le fonctionnement du système racinaire. Plusieurs études ont déjà montré que l'activité racinaire était réduite dans

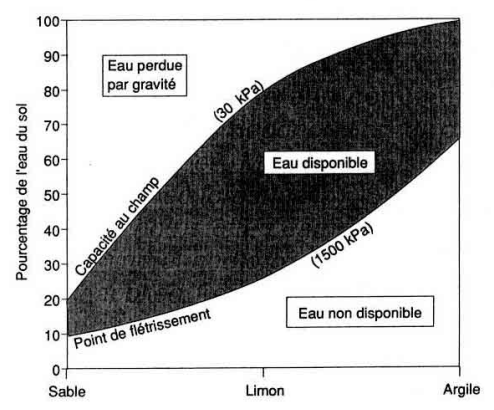

Fig 1. Disponibilité de l'eau du sol en fonction de la texture. 
des substrats froids $\left(0-10^{\circ} \mathrm{C}\right.$ ) (Tryon et Chapin, 1983; Lopushinsky et Kaufmann, 1984; Lieffers et Rothwell, 1986; Grossnickle, 1987). L'absorption d'eau (et de nutriments) y est réduite en raison de la diminution de la perméabilité des racines (Kaufmann, 1977) et d'une plus grande viscosité de l'eau (Cooper, 1973). Généralement, la croissance des racines augmente avec la température du sol jusqu'à un certain maximum $\left(20-30{ }^{\circ} \mathrm{C}\right.$ ) (Voorhees et al, 1981), au-delà duquel les températures s'avèrent létales pour les plantes $\left(45-60^{\circ} \mathrm{C}\right)$.

L'énergie utilisée pour le réchauffement du sol provient principalement de l'absorption du rayonnement solaire, qui ellemême dépend de la nature de la surface. D'abord fonction de sa couleur, le coefficient de réflexion (albedo) du sol est plus élevé pour les surfaces pâles (20-35\%) que pour les surfaces foncées $(8-15 \%)$ (Sellers 1969). Or, les horizons des sols forestiers sont souvent distinguables par leur couleur. Selon la couche mise à nu, le scarifiage est donc susceptible de modifier grandement la quantité d'énergie absorbée en surface. L'utilisation subséquente de l'énergie captée dépend de l'énergie nécessaire pour réchauffer le sol (capacité thermique) et de la facilité avec laquelle le sol transmet la chaleur (conductivité thermique), qui varient selon les proportions des divers constituants (particules minérales et organiques, eau et air). En gros, la conductivité thermique du sol augmente avec la densité apparente, puisque, de tous les constituants, la phase solide possède la plus grande conductivité. D'autre part, l'eau a une plus grande capacité thermique et est meilleur conducteur que l'air (Marshall et Holmes, 1988). Lorsque l'eau remplace l'air dans un sol relativement $\mathrm{sec}$, l'augmentation de la conductivité est plus importante que l'augmentation de la capacité thermique, ce qui signifie de plus grandes variations de température dans le profil. Pour un sol déjà humide, l'augmentation de la capacité thermique domine et les changements de température avec le temps et la profondeur sont faibles, d'autant plus qu'une fraction plus importante de l'énergie absorbée en surface sert à l'évaporation.

Dans la forêt boréale canadienne, la couche de matière organique, souvent épaisse, retarde le réchauffement estival du sol nécessaire au démarrage de la croissance racinaire des semis (Smith, 1988). Toute l'ampleur du phénomène n'est pas connue, même si l'on sait que le sol minéral peut demeurer gelé plusieurs jours après la disparition du couvert de neige (Prévost et al, 1989). Cet aspect est d'autant plus important que le régime thermique du sol influence grandement l'activité biologique et la minéralisation de la matière organique (Van Cleve et al, 1981; Salonius, 1983).

\section{Fertilité}

Divers éléments chimiques sont nécessaires pour la construction des tissus des plantes. Par la photosynthèse, le dioxyde de carbone est réduit en hydrates de carbone, qui servent de matière première pour des composés plus complexes. D'autres éléments doivent être puisés à même le sol, qui pour être fertile doit contenir les nutriments sous forme disponible pour la plante et en des proportions appropriées (Shear et al, 1946). Les micronutriments ( $\mathrm{Fe}, \mathrm{Mn}, \mathrm{Cu}, \mathrm{Zn}, \mathrm{Mo}, \mathrm{Cl}, \mathrm{B}$ ) sont disponibles en quantité adéquate dans la plupart des sols forestiers par suite de l'altération de la roche-mère, tandis que la disponibilité des macronutriments ( $N, P, K$, $\mathrm{Ca}, \mathrm{Mg}, \mathrm{S}$ ) dépend principalement de leur recyclage à l'intérieur de l'écosystème (Waring et Schlesinger, 1985). En milieu forestier, la couche de matière organique constitue de loin la source principale de 
nutriments du sol (Armson, 1977; Keeney, 1980). La décomposition des composés organiques complexes (cellulose, hemicellulose, lignine, protéines, sucres, composés secondaires) en des formes solubles d'éléments minéraux (minéralisation) s'y fait surtout par les bactéries et les champignons. La vitesse de décomposition est largement contrôlée par la température, l'humidité et la qualité du substrat. L'activité microbienne augmente avec la température tandis qu'elle varie de façon parabolique avec la teneur en eau (fig 2). De façon générale, on observe donc une stimulation marquée de la décomposition par l'augmentation de la température (Salonius, 1983), par l'humidification des sols secs ou l'aération des sols trop humides (Waring et Schlesinger, 1985). À l'extrême, la décomposition des sols inondés de la forêt boréale se trouve fortement retardée à la fois par le manque d'aération et les basses températures (Van Cleve et al, 1981).

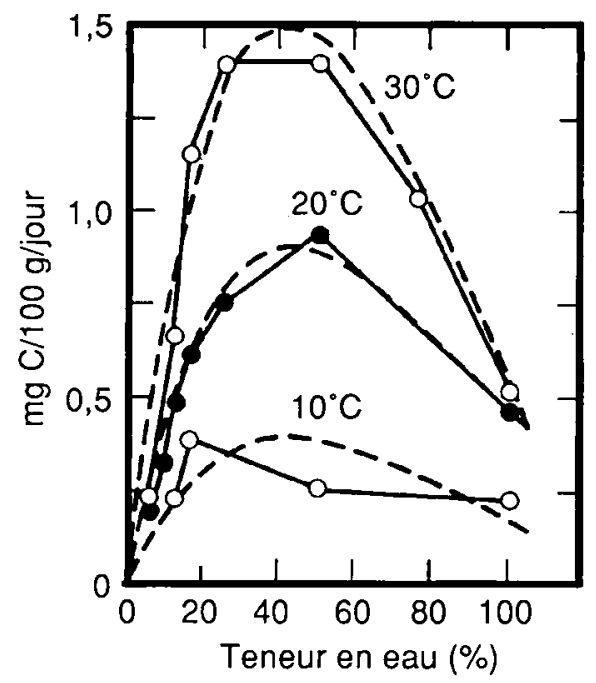

Fig 2. Relâchement de $\mathrm{C}$ sous forme de $\mathrm{CO}_{2}$ dû à l'activité microbienne en relation avec la température et la teneur en eau du sol (tiré de lno et Monsi, 1969; in: Waring et Schlesinger, 1985).

\section{Densité apparente}

La densité apparente du sol (masse anhydre/volume) est étroitement reliée aux autres propriétés physiques du sol (texture, structure, distribution des pores, contenu en matière organique) et influence elle-même plusieurs paramètres importants dans le système sol-plante (résistance mécanique à la croissance racinaire, régime humidité-aération, régimes thermique et nutritif du substrat). Puisqu'elle se détermine de façon relativement simple, la densité apparente est souvent utilisée à titre de variable synthèse pour évaluer le degré de perturbation des sols avant et après traitement.

En ce qui a trait à la croissance racinaire, l'augmentation de la densité pour une même texture signifie une diminution de la porosité totale, c'est-à-dire de l'espace disponible pour l'eau, l'air et les racines elles-mêmes (fig 3). Un niveau de compaction modéré (densité de 1,05 $\mathrm{Mg} \cdot \mathrm{m}^{-3}$ ) est caractérisé par la disparition de macropores, compensée en partie par la création de micropores (Greacen et Sands, 1980). Ceci se traduit par une meilleure rétention en eau, mais aussi principalement par une baisse de la capacité d'aération (fig 3), par rapport au sol moins dense. Une densité plus élevée (par ex : 1,25 et 1,45 Mg.m ${ }^{-3}$ ) entraîne une perte de micropores (Hill et Sumner, 1967), diminuant à la fois la capacité de rétention en eau et l'aération. À l'extrême, la capacité d'aération s'abaisse sous le seuil critique d'environ $10 \%$ du volume de sol généralement reconnu comme étant nécessaire pour l'activité racinaire (Vomocil et Flocker, 1961; Greacen et Sands, 1980). En plus des conditions asphyxiantes qu'ils présentent, les sols denses opposent une résistance mécanique à la croissance racinaire. Bien qu'il soit difficile de séparer l'effet de la résistance mécanique de ceux de la dis- 


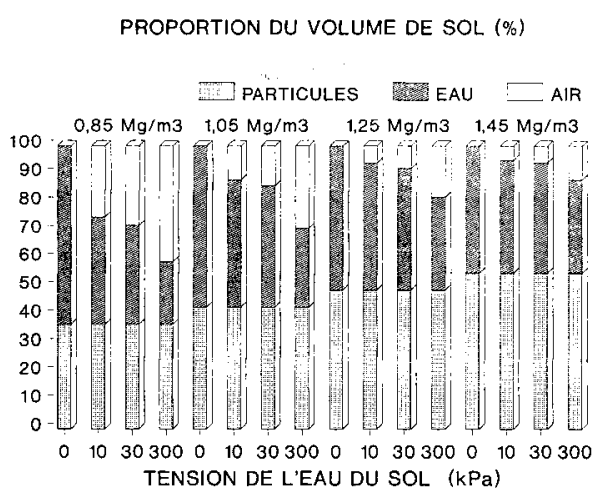

Fig 3. Proportion de particules solides, d'eau et d'air dans un sol sableux en fonction de la densité apparente $\left(\mathrm{Mg} / \mathrm{m}^{3}\right)$ et de la tension de l'eau du sol $(\mathrm{kPa})$. (reproduit de Prévost et Bolghari, 1990.)

ponibilité en eau et du manque d'aération, les études de compaction in vitro ont démontré que selon la texture des sols et l'essence considérée, la pénétration des racines est fortement compromise lorsque la densité apparente atteint 1,2 à 1,8 Mg.m-3 (tableau I).

Dans les sols forestiers, la densité apparente varie généralement de $0,2 \mathrm{Mg} \cdot \mathrm{m}^{-3}$ dans les horizons organiques à près de $1,9 \mathrm{Mg} \cdot \mathrm{m}^{-3}$ dans les sables grossiers (Pritchett e. Fisher, 1987). Certains dépôts, d'origine glaciaire ou riches en argile, sont naturellement denses (Raney et al, 1955). À l'état naturel, les couches profondes du sol (horizon C) ont généralement des densités apparentes plus élevées qu'en surface où l'horizon développé en place contient plus de matière organique. Toutefois, la machinerie lourde peut entraîner la compaction de la surface du sol lors des opérations forestières (Greacen et Sands, 1980). Sous des conditions favorisant l'accumulation d'une épaisse couche de matière organique, l'effet amortisseur de cette dernière a cependant été noté (Waster- lund, 1985; Corns, 1988). Ce phénomène pourrait s'appliquer à certains sites de la forêt boréale québécoise, où la compaction induite par la machinerie a reçu jusqu'à maintenant très peu d'attention.

\section{EFFETS DU SCARIFIAGE SUR LES PROPRIÉTÉS DU SOL}

\section{Disponibilité de l'eau}

Le scarifiage influence le régime hydrique du sol en changeant à la fois les pertes par évapotranspiration et les propriétés de rétention en eau du substrat. En diminuant les débris de coupe et la végétation compétitrice, le scarifiage augmente le rayonnement solaire et le vent dans l'entourage des semis, pour ainsi accroître la demande évaporante de l'air (Lanini et Radosevich, 1986) et entraîner une hausse de transpiration de la part des semis. Toutefois, l'apport d'eau au sol par les précipitations augmente significativement, puisque les débris et la strate arbustive ont une capacité d'interception importante, pouvant atteindre, par exemple, $30 \%$ des précipitations 4 ans après la coupe à blanc dans une sapinière laurentidienne (Prévost et Plamondon, 1987). De plus, en enlevant la végétation compétitrice, le scarifiage diminue les prélèvements pour la transpiration et contribue à conserver l'eau disponible pour les semis (Plamondon et al, 1980; Burger et Pritchett, 1988; Lanini et Radosevich, 1986). Ainsi, les plus grandes réserves en eau du sol comblent amplement la demande évaporante accrue (McMinn, 1984) et entraînent une diminution du stress hydrique des plants (Barber, 1984; Lanini et Radosevich, 1986). En ce sens, Muelder et al (1963) avaient noté que les semis de conifères n'étaient pas affectés par des demandes évaporantes élevées lorsque l'eau du sol était abondante. Lorsque les condi- 
Tableau I. Densité apparente du sol $\left(\mathrm{Mg} \cdot \mathrm{m}^{-3}\right)$ à laquelle la croissance racinaire des semis est nette- . ment réduite.

\begin{tabular}{|c|c|c|c|}
\hline Référence & Sol et densité testés & Essence & $\begin{array}{l}\text { ité apparente } \\
\left.M g . m^{-3}\right)\end{array}$ \\
\hline $\begin{array}{l}\text { Armson et } \\
\text { Williams (1960) }\end{array}$ & $\begin{array}{l}\text { Sable } \\
(1,02 \text { et } 1,45)\end{array}$ & Pinus resinosa Ait & 1,45 \\
\hline Berben (1973) & $\begin{array}{l}\text { Sable humique de l'horizon } B \\
\text { de profils podzoliques } \\
(0,9,1,2 \text { et } 1,5)\end{array}$ & $\begin{array}{l}\text { Pinus nigra Arnold } \\
\text { var Corsicana } \\
\text { Larix leptolepis Gord et } \\
\text { Pseudotsuga menziesii } \\
\text { Betula pendula Roth et } \\
\text { Alnus incana Moench }\end{array}$ & $\begin{array}{l}1,5 \\
1,2\end{array}$ \\
\hline Corns (1988) & $\begin{array}{l}\text { Argile limoneux lacustre } \\
(1,2,1,35 \text { et } 1,5) \\
\text { Loam argileux glaciaire } \\
(1,3,1,4 \text { et } 1,5) \\
\text { Loam sableux fluvial } \\
(1,3,1,4 \text { et } 1,5)\end{array}$ & 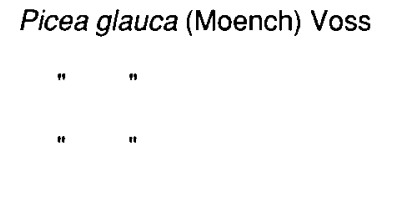 & $\begin{array}{l}1,5 \\
1,5 \\
1,4\end{array}$ \\
\hline $\begin{array}{l}\text { Foil et Ralston } \\
(1967)\end{array}$ & $\begin{array}{l}\text { Sable loameux } \\
(0,96 \text { à } 1,42) \\
\text { Loam } \\
(0,87 \text { à } 1,38) \\
\text { Argile } \\
(0,88 \text { à } 1,48)\end{array}$ & $\begin{array}{l}\text { Pinus taeda L } \\
\text { " } " \\
" \quad "\end{array}$ & $\begin{array}{l}1,33 \\
1,20 \\
1,31\end{array}$ \\
\hline Frisque (1977) & $\begin{array}{l}\text { Mélange sable, tourbe et terre } \\
\text { (15 densités de } 1,03 \text { à } 1,53)\end{array}$ & $\begin{array}{r}\text { Picea mariana (Mill) BSP } \\
\text { (Selon suiv } \\
\text { et croissar }\end{array}$ & $\begin{array}{l}1,25 \\
\text { ji de mortalité) } \\
\text { nce aérienne }\end{array}$ \\
\hline Heilman (1981) & $\begin{array}{l}\text { Loam } \\
(1,38,1,57 \text { et } 1,76) \\
\text { Loam sableux } \\
(1,38,1,57 \text { et } 1,76)\end{array}$ & $\begin{array}{c}\text { Pseudotsuga menziesii } \\
" ~ "\end{array}$ & $\begin{array}{l}1,57 \\
1,57\end{array}$ \\
\hline Minore et al (1969) & $\begin{array}{l}\text { Loam sableux } \\
(1,32,1,45 \text { et } 1,59)\end{array}$ & $\begin{array}{l}\text { Picea sitchensis, } \\
\text { Tsuga heterophylla, } \\
\text { Thuya plicata et Abies amabilis } \\
\text { Pseudotsuga menziesii, } \\
\text { Pinus contorta et Alnus rubra }\end{array}$ & $\begin{array}{l}1,45 \\
1,59\end{array}$ \\
\hline $\begin{array}{l}\text { Prévost et Bolghari } \\
\text { (1990) }\end{array}$ & $\begin{array}{l}\text { Sable de l'horizon } B \\
\text { de profils podzoliques } \\
(0,85,1,05,1,25 \text { et } 1,45)\end{array}$ & Picea mariana (Mill) BSP & 1,25 \\
\hline $\begin{array}{l}\text { Simmons et Pope } \\
\text { (1987) }\end{array}$ & $\begin{array}{l}\text { Loam limoneux } \\
(1,25,1,40 \text { et } 1,55)\end{array}$ & $\begin{array}{l}\text { Liriodendron tulipifera } \mathrm{L} \text { et } \\
\text { Liquidambar styraciflua } \mathrm{L}\end{array}$ & 1,55 \\
\hline Zisa et al (1980) & $\begin{array}{l}\text { Loam limoneux } \\
(1,2,1,4,1,6 \text { et } 1,8)\end{array}$ & $\begin{array}{l}\text { Pinus rigida Mill } \\
\text { Pinus nigra Arnold et } \\
\text { Picea abies (L) Karst } \\
\text { " }\end{array}$ & $\begin{array}{l}1,4 \\
1,6\end{array}$ \\
\hline
\end{tabular}


tions évaporantes sont extrêmes, le sol mis à nu peut toutefois s'assécher rapidement en surface (Sims, 1975; Morris et Pritchett, 1983) et empêcher la germination ou nuire considérablement à la survie des jeunes semis dont l'enracinement est encore superficiel.

On reconnaît généralement que les méthodes de scarifiage qui incorporent la matière organique au sol minéral produisent un mélange dont la rétention en eau et l'aération sont assurés à long terme (Orlander et al, 1990). Les sols à texture fine y acquièrent une meilleure aération par la création de macropores (Moehring, 1970), tandis que les sols à texture grossière bénéficient d'une meilleure rétention en eau (Pritchett et Fisher, 1987) par la formation d'agrégats. Notamment, le bris de capillarité occasionné par le scarifiage est susceptible de diminuer les pertes par évaporation en surface (Plamondon et al, 1980).

Dans les sols périodiquement inondés ou particulièrement compacts, l'intervention (drainage, scarifiage, mise en buttes) doit viser une meilleure aération afin de favoriser le développement racinaire, mais en conservant une bonne cohésion du substrat et un approvisionnement en eau suffisant. La création de microsites surélevés est une façon reconnue d'améliorer le drainage local et l'aération, tout en réduisant l'obstruction par la végétation avoisinante (Haeussler, 1989). En période sèche, le sommet des monticules est toutefois susceptible de s'assécher rapidement (McMinn, 1984; Orlander, 1986), puisqu'une plus grande surface est exposée à l'ávaporation (Bassman, 1989). Par exemple, des tensions matricielles variant entre environ 200 et $1300 \mathrm{kPa}$ ont été mesurées à $5 \mathrm{~cm}$ sous la surface après la mise en buttes dans un sol limoneux, alors qu'elles n'excédaient pas $500 \mathrm{kPa}$ à $12 \mathrm{~cm}$ sous la surface et $250 \mathrm{kPa}$ dans le sol minéral uniquement mis à nu (fig 4). D'autre part, malgré la rareté de données de ré- tention en eau en fonction de la position sur les monticules, il semblerait reconnu que les flancs moins élevés présentent des régimes hydriques plus favorables (Sutton, 1984), ayant une meilleure continuité capillaire avec le sol minéral sousjacent. Quoi qu'il en soit, la planification des travaux de scarifiage doit tenir compte du fait que les conditions extrêmes de sécheresse à la surface du sol, même passagères, peuvent décider du succès de régénération (naturelle ou artificielle). Elle doit intégrer les différentes propriétés du site susceptibles d'influencer le régime hydrique du sol (précipitations, température, rétention en eau du sol). Au Québec, aucune étude connue n'a examiné le régime hydrique ou l'enracinement en fonction de la position sur les microsites, des types de sols et du microclimat en surface.

\section{Régime thermique}

On note généralement une hausse de la température du sol après scarifiage (Malkonen, 1972; Dobbs et McMinn, 1973; Pla-

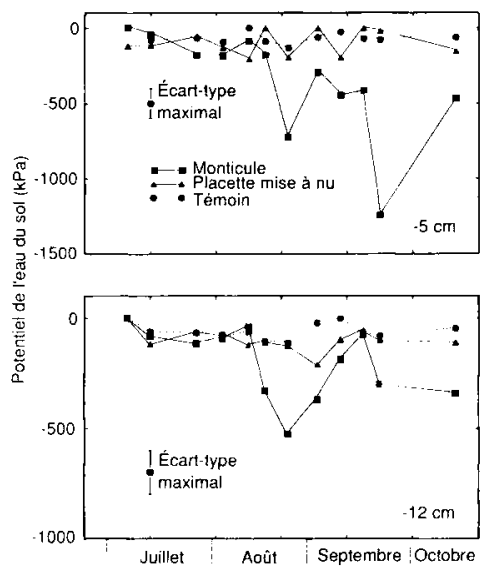

Fig 4. Potentiel de l'eau du sol à 5 et $12 \mathrm{~cm}$ sous la surface, en fonction du type de microsite durant la saison 1982 (tiré de Bassman, 1989). 
mondon et al, 1980; Palmgren, 1984; Bassman, 1989; Orlander et al, 1990). Deux phénomènes provoquent principalement cette hausse : (1) l'augmentation du rayonnement solaire direct au sol rendu possible par l'enlèvement des débris et de la végétation et (2) l'amélioration des propriétés thermiques du sol par la mise à nu de l'horizon minéral (enlèvement de la couche isolante) ou par l'incorporation de la matière organique au sol minéral. Les températures atteintes varient grandement entre les types de scarifiage utilisés. Généralement, la formation de monticules permet un meilleur réchauffement que la simple mise à nu du sol minéral, puisque les microsites surélevés (ou exposés au sud) reçoivent plus de rayonnement solaire (Orlander et al, 1990). Illustrant bien cet énoncé, les résultats de Bassman (1989), pour un site pluvieux situé en altitude, indiquent des températures ayant atteint $25{ }^{\circ} \mathrm{C}$ à $5 \mathrm{~cm}$ sous la surface des monticules alors qu'elles n'excédaient pas $15^{\circ} \mathrm{C}$ à la même profondeur au fond des microsites mis à nu. À l'extrême, sous insolation intense, les sols secs peuvent atteindre des températures létales pour la régénération. Des maxima dépassant $55^{\circ} \mathrm{C}$ près de la surface ont d'ailleurs été observés par Morris et Pritchett (1983) dans des sables fins sous le climat chaud de la Floride. De même, des températures excédant $50^{\circ} \mathrm{C}$ ont été enregistrées par Sims (1975) dans des sables à texture moyenne de la forêt boréale du Sud-Est du Manitoba. Cependant, les températures optimales pour la germination et la croissance racinaire sont suffisamment élevées (Heninger et White, 1974) pour que les niveaux atteints sous la surface du sol aient généralement un effet bénéfique (Nielson, 1974; McMinn, 1984).

La perturbation du couvert isolant s'accompagne aussi d'une hausse des écarts journaliers de température, principalement dans les couches de surface (Kauppila et
Lahde, 1974; Morris et Pritchett, 1983; Orlander, 1987); ce phénomène est susceptible d'amplifier le cycle gel - dégel associé au déchaussement des semis. En climat froid, dans un site de la forêt boréale québécoise (latitude $50^{\circ} \mathrm{N}$ ), la température à $1 \mathrm{~cm}$ sous la surface de microsites scarifiés a varié entre 0 et $36^{\circ} \mathrm{C}$ au cours des étés 1990 et 1991 , alors qu'elle a fluctué entre 3 et $13^{\circ} \mathrm{C}$ dans le sol recouvert d'humus (Prévost, données non publiées). Le scarifiage amplifie également les écarts dans le cycle annuel de la température du sol (fig 5), en accélérant le réchauffement printanier avec toutefois en contrepartie un gel automnal plus précoce. Cette modification du régime thermique devrait être particulièrement bénéfique dans le cas des sols froids de la forêt boréale.

\section{Fertilité}

Puisque l'horizon organique est le premier perturbé par le scarifiage, la disponibilité en minéraux peut être directement affectée. Diverses études ont montré que la mise à nu du sol, en enlevant les débris de coupe, la végétation compétitrice et la matière organique, cause une diminution significative des éléments nutritifs disponibles dans l'entourage des semis (Morris et Pritchett, 1983; Ezell et Arbour, 1985;

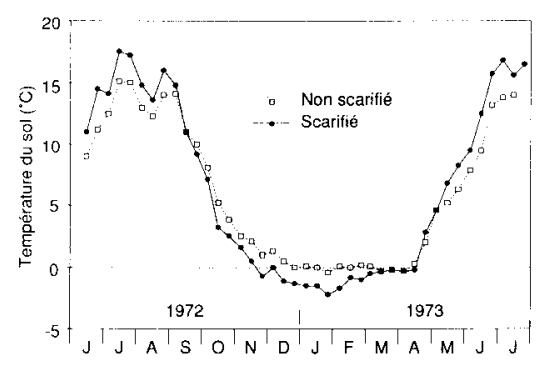

Fig 5. Effet du scarifiage sur la température moyenne du sol entre 5 et $60 \mathrm{~cm}$ de profondeur (tiré de Plamondon et al, 1980). 
Stransky et al, 1985; Tuttle et al, 1985a; Vitousek et Matson, 1985). Même en présence de grandes réserves d'azote dans le sol (matières humiques), l'approvisionnement de matière organique fraîche doit être maintenu puisqu'elle représente, après minéralisation, la source première d'azote utilisée par la plante, d'autant plus que l'accumulation des réserves peut indiquer la présence d'azote sous forme non disponible (Hall, 1984). Cependant, en climat froid, le faible taux de minéralisation limite déjà la disponibilité de l'azote. Ainsi, les pertes nutritionnelles résultant de l'enlèvement de la matière organique de surface seraient plus que compensées à court terme par les effets bénéfiques sur le régime thermique (augmentation de l'activité racinaire et de la minéralisation) des sols à texture moyenne (McMinn, 1984). Toutefois, les études suggèrent que la conservation des nutriments contenus dans la matière organique pourrait être bénéfique surtout à long terme (Moehring, 1970; Hall, 1985; Needham, 1986), pour combler les besoins nutritionnels du futur peuplement.

Les techniques de scarifiage qui mélangent la matière organique au sol minéral permettent de conserver une source adéquate de nutriments pour les semis (McMinn, 1984), tout en favorisant la minéralisation (Burger et Pritchett, 1984; Palmgren, 1984; Orlander et al, 1990) par des conditions favorables de température et d'humidité du sol. Cette minéralisation accrue ne correspond toutefois pas nécessairement à une augmentation des prélèvements par la masse racinaire, mais est susceptible d'augmenter les pertes par lessivage car elle se produit au stade où la demande totale en azote par la biomasse est minimale (CEMAGREF, 1976). Même si certains processus peuvent diminuer le lessivage des nitrates des sols perturbés (immobilisation, baisse de nitrification laissant l'azote sous forme d'ammonium qui est moins mobile), ils ne suffisent généralement pas à empêcher complètement les pertes en l'absence de prélèvements importants par la végétation (Vitousek et al, 1979). L'accélération du lessivage des éléments nutritifs suite à la forte perturbation du sol pourrait donc annuler les effets bénéfiques observés à court terme (Gagnon, 1969; Haines et al, 1975; McClurkin et Duffy, 1975; Morris et Pritchett, 1983). Toutefois, les études sur la productivité des sites à long terme sont encore trop récentes pour permettre de tirer des conclusions (Orlander et al, 1990). Face à la possibilité d'un appauvrissement à long terme, la planification des travaux de scarifiage devrait pouvoir s'appuyer sur des critères reflétant la susceptibilité des sites au lessivage des éléments nutritifs (érodibilité, pente, épaisseur de la matière organique, fréquence et intensité des précipitations). Dans les conditions de la forêt boréale québécoise, où la minéralisation de la matière organique est lente, aucune étude n'a encore examiné l'effet du scarifiage sur le potentiel nutritif des sites, que ce soit à court ou à long terme.

\section{Densité apparente}

Les recherches sur des sols ayant subi une compaction ont démontré que les techniques de scarifiage mélangeant la matière organique au sol minéral pouvaient ramener la densité apparente des horizons de surface $(0-20 \mathrm{~cm})$ à leur niveau d'avant coupe (Gent et al, 1984; Gent et Ballard, 1985). De plus, il est reconnu que la formation de microsites surélevés, en ameublissant le sol, réduit sa densité apparente et augmente sa porosité (Orlander et al, 1990). Par ailleurs, sur des sols compactés (d'origine naturelle ou induite par la machinerie), les méthodes qui enlèvent la couche de matière organique sans perturber le sol minéral risquent d'exposer 
un horizon beaucoup plus dense (Tuttle et al, 1985a; Ross et al, 1986), susceptible d'être peu propice à l'enracinement (résistance mécanique, manque d'aération). À l'inverse, un scarifiage profond peut permettre d'améliorer un profil compacté en augmentant la porosité totale et l'aération (Moehring, 1970), pour favoriser une pénétration racinaire en profondeur. De façon générale, le scarifiage laisse une base de sol ferme au fond des sillons, un r rélange détassé matière organique - sol minéral au sommet des monticules et un sol de densité intermédiaire sur l'épaulement des monticules. Les niveaux de densité créés en fonction de la profondeur et selon la position sur les microsites restent à être étudiés en relation avec les caractéristiques des sites (matériel parental, épaisseur de la matière organique, sol). L'aptitude à l'enracinement et la croissance de diverses essences en fonction des microsites dojvent également être examinées pour les conditions de la forêt boréale québécoise.

\section{EFFETS DU SCARIFIAGE SUR L'ÉTABLISSEMENT ET LA CROISSANCE DES SEMIS}

\section{Ensemencement}

De nombreux auteurs, répertoriés par Place (1955), ont depuis longtemus désigné le sol minéral comme étant un excellent lit de germination pour les essences résineuses, principalement pour l'épinette rouge (Picea rubens Sarg), l'épinette blanche (Picea glauca Moench Voss), l'épinette noire (Picea mariana Mill BSP) et le sapin baumier (Abies balsamea $L$ Mill). On note un réchauffement rapide et une bonne rétention en eau du sol (l'humidité en surface étant le facteur prépondérant pour la germination) comme étant les qualités premières de ce substrat. Plus récem- ment, les recherches ont montré que l'exposition du sol minéral par le scarifiage améliorait la régénération naturelle du pin à encens (Pinus taeda L) dans le climat chaud et humide du Sud des États-Unis (Haines et al, 1975; Cain, 1987), et du pin gris (Pinus banksiana Lamb) dans le climat généralement froid et humide de la forêt boréale du Québec (Demers, 1978) et de l'Ontario (Mattice et McPhee, 1979). Également en forêt boréale, le scarifiage a nettement amélioré la germination de l'épinette noire (Frisque, 1977; Jeglum, 1980; Orynik, 1985). En Alberta, les expériences portant sur le pin Lodgepole (Pinus contorta Dougl) et l'épinette blanche ont aussi montré le succès du scarifiage pour promouvoir la régénération naturelle dans des sites à haute altitude (Wright, 1985).

De façon générale, on mentionne que le scarifiage visant à favoriser la régénération naturelle doit être plus intense que pour la plantation (Brown, 1977; Robinson, 1987; Orlander et al, 1990). En effet, pour que la régénération naturelle soit bien distribuée, le nombre de bons microsites créés doit être supérieur afin de compenser la répartition aléatoire des graines. En ce sens, le scarifiage par sillons continus serait préférable au traitement par placettes (Orlander et al, 1990). Dans des cas extrêmes, une régénération trop abondante peut être obtenue si la quantité de microsites réceptifs est excessive (Jeglum, 1987). Quoi qu'il en soit, le nombre de microsites requis dépend de la disponibilité des semences, elle-même fonction du nombre de semenciers laissés sur pied et de l'abondance de la production semencière. Pour des coupes par bandes dans l'épinette noire, Jeglum (1987) a obtenu une distribution des semis de $60 \%$ en produisant $5 \%$ de microsites réceptifs dans des bandes de $20 \mathrm{~m}$ de largeur alors qu'il en fallait entre 15 et $35 \%$ pour des bandes de $80 \mathrm{~m}$. D'autre part, les conditions permettant une bonne germination ne sont pas nécessai- 
rement propices à la croissance juvénile des senis. Les couches profondes du sol contiennent peu de nutriments, d'autant plus qu'une perturbation intense risque d'entraîner le lessivage. Malheureusement, peu d'études connues ont suivi la croissance des semis issus d'ensemencement (naturel ou artificiel) après scarifiage.

\section{Plantation}

II est reconnu que les semis plantés sont affectés par un choc de plantation, souvent causé par un stress hydrique (Aussenac et El Nour, 1986). Pour diverses raisons (dessèchement des plants, mauvais contact racine-sol, destruction des radicelles), la capacité réduite d'absorption d'eau empêche le bon déroulement des fonctions biologiques. Les études démontrent qu'à peu près toutes les formes de scarifiage permettent un meilleur établissement des plants et que, sous cet aspect, les méthodes s'équivalent au cours des premièr যs années (Derr et Mann, 1970; Haines et al, 1975; Stafford et al, 1985; Wilhite et McKee, 1985; Ross et al, 1986; Wittwer et al, 1986; Orlander, 1987). Lors du choc de plantation, de bonnes conditions de température, de structure et de rétention en eau du sol améliorent l'efficacité du système racinaire et favorisent la reprise du mouvement de l'eau dans les plants.

Diverses études font état d'une reprise rapide de la croissance des plants à la suite du bon établissement après scarifiage. Dans un sol sableux de la forêt boréale québécoise, le taux de croissance des 5 premières années de plants de pin gris a été nettement supérieur en terrain labouré qu'en sol non remanié (Doucet, 1986). Au Wisconsin, Rawinski et al (1980) avaient montré l'effet positif de l'incorporation de la matière organique au sol minéral loameux dès la croissance juvé- nile chez cette essence. Les méthodes mécaniques de préparation de terrain ont nettement augmenté la croissance du pin à encens au cours des 3-5 premières années dans les Piedmonts de Caroline du Sud (Stafford et al, 1985) et dans une région du Texas aux étés chauds et humides (Stransky et al, 1985). Bien que Shultz (1975) estime que l'effet bénéfique subsiste plusieurs années chez les différents pins du Sud des États-Unis, certains doutes demeurent quant à l'effet sur la récolte finale (Haines et al, 1975; Morris et Pritchett, 1983).

D'autres études ont montré que le succès d'établissement de plantations sur des sites scarifiés n'assurait pas nécessairement la bonne croissance des plants. Ainsi, malgré des taux de survie élevés (80-90\%) avec tous les types de scarifiage 1-5 ans après plantation sur des sols de différentes textures dans le Sud des ÉtatsUnis, la croissance du pin à encens n'a été meilleure que sur les microsites les plus remaniés et surélevés dans les hautes terres des côtes d'Alabama (Tuttle et al, 1985b) et dans la basse plaine côtière de Caroline du Sud (Wilhite et McKee, 1985). En région montagneuse d'Oklahoma (Wittwer et al, 1986) et sous le climat chaud et humide de la Louisiane (Slay et al, 1987), les meilleurs résultats ont cependant été obtenus lorsque la végétation compétitrice était la mieux contrôlée à l'aide d'herbicides après scarifiage. Une croissance limitée de conifères après scarifiage sans autre traitement a aussi été notée dans les monts Cascades d'Orégon (Ross et al, 1986) et en Nouvelle-Zélande (Ballard, 1978). En Australie, l'enlèvement des débris de coupe et de la matière organique avait grandement aidé l'établissement du pin radiata (Pinus radiata $D$ Don) en haute altitude (1 $200 \mathrm{~m})$, sans toutefois améliorer la croissance des 2 premières années par rapport au sol non traité (Hall, 1985). L'auteur, attribue la stagnation des semis à la 
perte de nutriments encourue par l'enlèvement de l'horizon de surface.

L'expérience suédoise en matière de scarifiage désigne la formation de monticules comme les microsites les plus favorables pour le pin Lodgepole, le pin sylvestre (Pinus sylvestris) et l'épinette de Norvège (Picea abies). Sur des sols sableux de la forêt boréale de l'Ontario, la mise en buttes a provoqué des taux de mortalité de $10-50 \%$ chez le pin gris planté au sommet des monticules et une meilleure performance (93-99\% de survie) sur l'épaulement, moins sujet à l'assèchement (Sutton, 1984). Considérant globalement le taux de survie, la croissance en hauteur et la croissance en diamètre, la localisation sur l'épaulement paraiss ait toujours la plus favorable pour cette essence, 5 ans après plantation (Sutton, 1987). Ayant été testée sur le même dispositif, l'épinette noire a eu des performances supérieures sur le sommet des monticules, malgré un été plus sec que la normale. Se basant sur des observations s'échelonnant sur plus de 10 ans après plantation en Suède, Orlander (1987) a noté que les plants sur monticules étaient supérieurs aux plants dans les sillons de disques ou de charrues, tant pour le taux de survie que pour la croissance. Les résultats des premières années n'avaient permis de détecter que de faibles différences entre les microsites, ce qui indique la nécessité d'un suivi à long terme pour évaluer la performance des plants suite au scarifiage.

Concernant l'utilisation et le développement de l'équipement de préparation de terrain, McMinn (1985) a souligné l'importance de la durée de la période d'observations pour l'interprétation des résultats. Après 10 ans, le suivi de plants d'śpinette blanche et de pin Lodgepole a finalement indiqué la supériorité de rasages répétés de la compétition sur le mixage du sol dans un site nordique de la Colombie Britannique, bien que les 2 traitements mon- traient des résultats de croissance équivalents après 5 ans. À l'inverse, sur un parterre de coupe d'une érablière à bouleau jaune dans la forêt feuillue du Québec, les secteurs scarifiés indiquaient une augmentation de $50 \%$ de la croissance des plants de bouleau jaune (Betula alleghaniensis Britton) dès la première année. Toutefois, les résultats quinquennaux ont par la suite montré que le scarifiage n'avait finalement pas amélioré la croissance des plants mais avait provoqué une plus grande agressivité de la compétition (Robitaille, 1978). Une telle augmentation de la compétition pour les semis plantés a également été rapportée par Durand et al (1988) à la suite d'un scarifiage de sols argileux dans la forêt boréale du Nord-Ouest québécois. Les auteurs y ont noté que la réduction de la densité des arbres feuillus compétiteurs s'était surtout traduite par une hausse importante du recouvrement des autres groupes d'espèces compétitrices (arbustes, framboisiers, herbacées). En climat humide subtropical, la diversité spécifique des compétiteurs avait également augmenté à la suite de la coupe à blanc et de la préparation de terrain dans les forêts de pin des bas-fonds boisés de la Floride (Swindel et al, 1984). Sur un parterre de coupe d'une forêt de sapin baumier et d'épinette noire de l'Est du Québec, la densité des espèces de début de succession, quant à elle, a augmenté avec l'intensité du scarifiage (Jobidon, 1990).

Lors de la planification des travaux de préparation de terrain, il faut garder à l'esprit que la végétation peut réoccuper un site par différents mécanismes (sexué ou végétatif) au cours du stade d'établissement d'un peuplement (Oliver, 1981). Par exemple, les rejets de souches permettent à certaines essences d'envahir un site rapidement et d'occuper l'espace de croissance avant les espèces désirables. La germination de semences enfouies dans le sol et ramenées en surface peut aussi pro- 
voquer un envahissement d'espèces qui étaient absentes avant la perturbation du milieu. À cet effet, les recherches ont montré que la banque séminale du sol pouvait contribuer de façon significative à la régénération arbustive après perturbation (Roberts, 1981; Morgan et Neuenschwander, 1988). Selon le type de préparation de terrain et l'intensité appliquée, le traitement pourra donc favoriser l'un ou l'autre des modes de régénération et influencer grandement la structure et la dynamique du peuplement. II importe donc d'identifier les propriétés du site pouvant indiquer les possibilités d'invasion des compétiteurs. À cet effet, l'organisation de l'information doit s'appuyer sur une classification écologique pour être utilisable de façon pratique et adéquate.

\section{PERSPECTIVES DE RECHERCHES}

À ce jour au Canada, les travaux de recherches en matière de scarifiage ont surtout été effectués en Ontario (Sutton, 1987) et en Colombie Britannique (Hedin, 1989; McMinn, 1989), alors qu'au Québec, les résultats des recherches dans le domaine sont quasi inexistants malgré une utilisation toujours accrue de cette technique. La presque totalité des travaux québécois de scarifiage visaient, en 1986, l'établissement de plantations (Fortin et Im, 1987), principalement d'épinettes noires $(55 \%)$, de pins gris $(20 \%)$ et d'épinettes blanches (14\%). On reconnaît généralement que le scarifiage peut améliorer la survie de ces essences après plantation. Toutefois, les effets sur les propriétés des différents sols et sur la croissance juvénile en fonction du type de scarifiage ou de la position sur les microsites doivent être étudiés. Les études doivent être planifiées de façon à compléter ou confirm ər les résultats obtenus ailleurs dans des conditions comparables.
En raison des conditions météorologiques particulières et de la courte durée de la saison de croissance, le régime thermique du sol ainsi que le microclimat en surface sont considérés comme des facteurs limitatifs dans les régions nordiques (Smith, 1988). Ils doivent donc être l'objet d'une attention spéciale après un scarifiage. Les quelques résultats disponibles au Québec indiquent également que la végétation compétitrice est susceptible de profiter de la création de bons microsites autant, sinon plus, que les essences désirables (Robitaille, 1978; Durand et al, 1988). La perspective de risquer une dégradation des sites à plus ou moins long terme pour assurer un bon établissement des semis devrait à elle seule motiver la mise en place d'études intensives visant à identifier les traitements appropriés. L'analyse des résultats devrait faire le bilan des effets positifs et négatifs en tenant compte de l'interaction entre les conditions du sol, la réaction de la compétition et la croissance à long terme, pour déterminer l'impact du scarifiage sur la productivité réelle du site (McNabb et Campbell, 1985). En outre, afin de comprendre la réaction des semis (Margolis et Brand, 1990), des études visant à connaître le comportement écophysiologique des espèces sous aménagement seront nécessaires (Aussenac et Lévy, 1983).

Selon les projections pour les 10 prochaines années (Bolghari et al, 1986), les activités forestières de récolte (225 000 ha/an) se dérouleront principalement dans les pessières $(52 \%)$, les sapinières $(25 \%)$, les pineraies grises (4\%) et les forêts mélangées à dominance résineuse (19\%). Les pinèdes grises sont particulièrement propices à la plantation. Ainsi, les efforts de recherche sur cette essence devraient viser une meilleure reprise des plants. Toutefois, compte tenu qu'une hausse de température à la surface du sol serait susceptible de favoriser l'ouverture des cônes, 
on pourrait aussi chercher à améliorer l'ensemencement naturel de cette espèce. Les sapinières, quant à elles, montrent habituellement une abondance de régénération préétablie et ne présentent pas ou peu de problèmes de régénération naturelle. D'autre part, une partie des pessières et des peuplements mélangés à dominance résineuse présentent une régénération préexistante adéquate (Ruel, 1989). Lorsque la régénération est insuffisante, ces peuplements peuvent être remis en production par la plantation. Toutefois, l'expérience a montré que certains sites offrent également la possibilité de se régénérer par semences après coupe. Dans un tel contexte, la coupe avec protection de la régénération préétablie et la coupe à blanc modifiée (bandes, trouées, îlots semenciers) sont des méthodes qui améliorent la régénération par voie naturelle. Dans les parterres de coupe avec réserve de semenciers, la qualité du lit de germination peut être améliorée par le scarifiage, une opération qui s'avère de toute façon nécessaire pour la plantation. Cependant, les méthodes les plus souhaitables, ainsi que les niveaux d'intensité appliqués, restent à déterminer en relation avec les caractéristiques des sites. En considérant que, la plupart du temps, l'opération de scarifiage s'impose quelle que soit la méthode de régénération préconisée, il serait intéressant de comparer la performance à long terme de la régénération provenant d'ensemencement (naturel ou artificiel) avec celle de plants, en fonction du type de scarifiage.

Au Québec, la pratique actuelle en matière de régénération forestière favorise la régénération naturelle, mais lorsque le reboisement s'avère nécessaire, le scarifiage est largement pratiqué. Or, les coûts totaux sont élevés (production de plants, scarifiage et plantation) et la possibilité de régénérer de façon naturelle une partie des aires coupées en modifiant l'opération de scarifiage doit être envisagée.

\section{REMERCIEMENTS}

L'auteur tient à remercier les Drs HA Bolghari et $R$ Doucet du Service de la recherche appliquée, ainsi que les Drs C Camiré et AP Plamondon de la Faculté de foresterie et géomatique de l'Université Laval pour la révision de cet article. Le travail de dactylographie de Mmes S Bourassa et $\mathrm{N}$ Langlois a également été grandement apprécié.

\section{RÉFÉRENCES}

Armson KA (1977) Forest soils. Properties and processes. Univ Toronto Press, Toronto, Ontario, Canada

Armson KA, Williams JRM (1960) The root development of red pine (Pinus resinosa Ait) seedlings in relation to various soil conditions. For Chron 36, 14-17

Aussenac G, Lévy G (1983) Influence du dessèchement du sol sur le comportement hydrique et la croissance du chêne pédonculé (Quercus pedonculata Ehrl) et du frêne (Fraxinus excelsior L) cultivés en cases de végétation. Ann Sci For 40, 251-264

Aussenac G, El Nour M (1986) Reprise des plants et stress hydrique. Rev For $\mathrm{Fr}$ XXXVIII, 264-270

Ballard R (1978) Effect of slash and soil removal on the productivity of second growth radiata pine on a pumice soil. $N Z J$ For Sci 8, 248258

Barber HW (1984) Effects of site preparation on survival and moisture stress of interior Douglas - fir seedlings planted in grass. Tree Planters' Notes, Fall, 7-10

Bassman JH (1989) Influence of two site preparation treatments on ecophysiology of planted Picea engelmannii $\times$ glauca seedlings. $J$ Can Rech For 19, 1359-1370

Becker M (1977) Contribution à l'étude de la transpiration et de l'adaptation à la sécheresse des jeunes plants résineux. Exemple de trois sapins du pourtour méditerranéen (Abies alba, A nordmanniana, A numidica). Ann Sci For 34, 137-158

Becker M, Lévy G (1983) Influence d'un dessèchement du sol sur la nutrition minérale de 
jeunes plants de résineux. Ann Sci For 40, 325-336

Berben JC (1973) Influence de la densité du sol et des précipitations sur la croissance et le développement radiculaire de quelques espèces forestières. Bull Soc Roy For Belg 80 , 377-401

Bolghari HA, Doucet R, Martel J (1986) Programme de remise en fonction de la forêt. Volet : document de support relatif au renouvellement des forêts résineuses du Québec par la régénération naturelle. Min Éner Res Québec Serv Trait Sylv

Brown G (1977) Site preparation. In: Proc plantation establishment Symp. Kirkland Lake, Ontario, Canada, 21-23 September 1977. For Can Great Lakes For Res Cent Symp Proc 0-P-5, 46-48

Burger JA, Pritchett WL (1984) Effects of clearfelling and site preparation on nitrogen mineralization in a southern pine stand. Soil Sci Soc $f m J 48,1432-1437$

Burger JA, Pritchett WL (1988) Site presentation effects on soil moisture and available nutrients in a pine plantation in the Florida flatwoods. For Sci 34, 77-87

Cain MD (1987) Site preparation techniques for establishing natural pine regeneration on small forest properties. South J Appl For 11, 41-45

CEMAGREF (1976) Techniques de reboisement. Réflexions générales - conception. Min Agric France Grp Tech For Div Tech Rebois Note Tech 47

Cooper AJ (1973) Root temperature and plant growth. Commonw Agric Bureaux, Res Rev 4

Corns IGW (1988) Compaction by forestry equipment and effects on coniferous seedling growth on four soils in the Alberta foothills. J Can Rech For 18, 75-84

Demers A (1978) Évaluation des essais opérationnels de scarifiage et d'ensemencement de bûchés de forêts de pin gris dans les bassins des rivières Gatineau et St-Maurice. In : Atelier de travail sur l'aménagement du pin gris. Min Terres For Québec, 81-117

Derr HJ, Mann WF (1970) Site preparation improves growth of planted pines. USDA For Serv Res Note SO-106
Dobbs RC, McMinn RG (1973) The effects of site preparation on summer soil temperature in spruce fir cutovers in the British Columbia interior. For Can Bi-Month Res Notes 29, 6-7

Doucet R (1986) Effet du labour sur la croissance d'une plantation de pin gris. Min Éner Res Québec Note Rech For 31

Durand F, Bergeron Y, Harvey B (1988) Effets de la préparation de terrain sur le type et l'abondance des espèces végétales compétitrices dans le canton d'Hébécourt, Abitibi. Min Éner Res Québec Serv Amélioration, Région Abitibi-Témiscamingue

Ezell AW, Arbour SJ (1985) Long-term effects of scalping on organic matter content of sandy forest soils. Tree Planters' Notes, Summer, 13-15

Foil RR, Ralston CW (1967) The establishment and growth of Loblolly pine seedlings on compacted soils. Soil Sci Soc Am Proc 31, 565-568

Fortin Y, Im PC (1987) Statistiques concernant le reboisement et les travaux connexes réalisés dans les forêts publiques 1986-1987. Min Éner Res Québec Serv Régén For

Frisque $G$ (1977) Régénération naturelle de l'épinette noire. Thèse de doctorat, Université Laval Québec, Québec, Canada

Fryk J (1986) Adapted site preparation in Sweden. Skogsarbeten Res 5

Gagnon JD (1969) Soil improvement trials using scarification and fertilization in stagnant white spruce plantations, Quebec, Canada. Plant Soil 30, 23-33

Gent JA, Ballard R (1985) Impact of intensive forest management practices on the bulk density of lower coastal plain and Piedmont soils. South J Appl For 9, 44-48

Gent JA, Ballard R, Hassan AE, Cassel DK (1984) Impact of harvesting and site preparation on physical properties of Piedmont forest soils. Soil Sci Soc Am J 48, 173-177

Greacen EL, Sands R (1980) Compaction of forest soils. A review. Aust J Soil Res 18, 163189

Grossnickle SC (1987) influence of flooding and soil temperature on the water relations and morphological development of cold-stored black spruce and white spruce seedlings. J Can Rech For 17, 821-828 
Haeussler S (1989) Mounding for site preparation. For Can and British Columbia Min For FRDA Memo 100

Haines LW, Maki TE, Sanderford SG (1975) The effect of mechanical site preparation treatments on soil productivity and tree (Pinus taeda $L$ and $P$ elliottii Engelm var e/liottii) growth. In: Forest soils and forest land management (B Bernier et $\mathrm{CH}$ Winget, eds). Presses Univ Laval, Sainte-Foy, Québec, Canada, 379-395

Hall M (1984) Establishment of radiata pine on a high altitude second rotation site. I. Effect of site preparation on nutrient capital. Aust For 47, 194-198

Hall M (1985) Establishment of radiata pine on a high altitude second rotation site. II. Effect of site preparation on early survival and growth. Aust For 48, 79-83

Hedin IB (1986) Mechanical site preparation trials in coastal British Columbia. For Eng Res Inst Can Tech Rep TR-69

Hedin IB (1989) Inverted humus mounds: followup field assessments. For Eng Res Inst Can Spec Rep RS-58

Heilman $P$ (1981) Root penetration of Douglasfir seedlings into compacted soils. For Sci 27 , 660-666

Heninger RL, White DP (1974) Tree seedling growth at different soil temperatures. For $\mathrm{Sci}$ 20, 363-367

Hill JNS, Sumner ME (1967) Effect of bulk density on moisture characteristics of soils. Soil Sci 103, 234-238

Ino $Y$, Monsi $M$ (1969) An experimental approach to the calculation of $\mathrm{CO}_{2}$ amount evolved from several soils. Jpn J Bot 20 , 153-188

Jeglum JK (1980) Strip cutting in shallow-soil upland black spruce near Nipigon, Untario. I. Study establishment and site conditions. For Can Great Lakes For Cent Inf Rep O-X-315

Jeglum JK (1987) Alternate strip clearcutting in upland black spruce. II. Factors affecting regeneration in first-cut strips. For Chron 63, 439-445

Jobidon R (1990) Short-term effect of three mechanical site preparation methods on species diversity. Tree Planters' Notes, Fall, 39-42
Kaufmann MR (1977) Soil temperature and drying cycle effects on water relations of Pinus radiata. J Can Bot 55, 2413-2418

Kauppila A, Lahde $E$ (1974) On the effects of soil treatments on forest soil properties in north Finland. Folia For (Helsinki) 230

Keeney DR (1980) Prediction of soil nitrogen availability in forest ecosystems: a literature review. For Sci 26, 159-171

Kramer PJ (1962) The role of water in tree growth. In: Tree growth (TD Kozlowski, ed). The Ronald Press Co, New York, USA, 171182

Lanini WT, Radosevich JR (1986) Response of three conifer species to site preparation and shrub control. For Sci 32, 61-77

Lévy G (1968) Importance des propriétés du sol pour l'enracinement de Picea excelsa et de Pinus silvestris. Ann Sci For 25, 157-188

Lieffers VJ, Rothwell RL (1986) Effects of depth of water table and substrate temperature on root and top growth of Picea mariana and Larix laricina seedlings. J Can Rech For 16 , 1201-1206

Lopushinsky N, Kaufmann MR (1984) Effects of cold soil on water relations and spring growth of Douglas-fir seedlings. For Sci 30, 628-634

Malkonen E (1972) Some aspects concerning cultivation of forest soil. Folia For (Helsinki) 137

Margolis HA, Brand DG (1990) An ecophysiological basis for understanding plantation establishment. J Can Rech For 30, 375-390

Marsha!l TJ, Holmes JW (1988) Soil physics. Cambridge Univ Press, Cambridge, England

Mattice CR, McPhee HG (1979) Mechanized row seeding of Jack pine. For Can Great Lakes For Res Cent Inf Rep O-X-296

McClurkin DC, Duffy PD (1975) Evaluating impact of forest site preparation on soil and water quality in the US gulf coastal plain. In: Forest soils and forest land management $(B$ Bernier et $\mathrm{CH}$ Winget, eds). Presses Univ Laval, Sainte-Foy, Québec, Canada, 315-321

McMinn RG (1984) Mechanical site treatment prescriptions should be based on site specific knowledge. In: Proc symp mechanization of silvic: increasing quality and productivity. Thunder Bay, Ont, Canada, 17 September 1984; Can Pulp Pap Assoc 21-25 
McMinn RG (1985) Use and development of site preparation equipment. In: Proc mechanized silv workshop. Edmonton, Alberta, Canada, 29 February -2 March 1984. For Can Northern For Res Cent Inf Rep NOR-X-272, 2-7

McMinn RG (1989) Mechanical site preparation options in north central British Columbia. In: Proc northern silvic committee workshop. Prince George, British Columbia, Canada, 23 February 1988. For Can and British Columbia Min For FRDA Rep 030, 86-88

McNabb DH, Campbell RG (1985) Quantifying the impacts of forestry activities on soil productivity. In: Proc 1985 SAF national convention, Fort Collins, Colorado, USA, 28-31 July 1985, 116-120

Minore D, Smith CE, Woolard RF (1969) Effects of high soil density on seedling root growth of seven north-western tree species. USDA For Serv Res Note PNW-112

Moehring DH (1970) Forest soil improvement through cultivation. $J$ For $68,328-331$

Morgan P, Neuenschwander LF (1988) Seedbank contributions to regeneration of shrub species after clear-cutting and burning. J Car Bot 66, 169-172

Morris LA, Pritchett WL (1983) Effects of site preparation on Pinus eliottii - Pinus palustris flatwoods forest soil properties. In: Proc IUFRO Symp For Site Continuous Prod. USDA For Serv Gen Tech Rep PNW-163, 243-251

Muelder DW, Tappeiner TC, Hansen JH (1963) Measurement of potential evapotranspiration rates in ecology and silviculture with particular reference to the Piché atmometer. $J$ For $61,840-845$

Needham TD (1986) Factors affecting Loblolly pine growth following site preparation. Thèse de Ph D Virginia Polytech Inst State Univ, Blacksburg, Virginia, USA

Nielson KF (1974) Roots and shoots temperatures. In: The plant root and its environment (EW Carson, ed) Univ Press Virginia, Charlotteville, Virginia, USA, 193-333

Oliver CD (1981) Forest development in North America following major disturbances. For Ecol Manage 3, 153-168

Orlander G (1986) Effect of planting and scarification on the water relations in planted seedlings of Scots pine. Stud For Suec 173
Orlander G (1987) Effects of site preparation on the development of planted seedlings in northern Sweden. In: Proc IUFRO Symp. Helsin$k i$, Finland

Orlander G, Gemmel P, Hunt J (1990) Site preparation: a Swedish overview. For Can and British Columbia Min For FRDA Rep 105

Orynik RJ (1985) Mechanized silviculture at Prince Albert pulpwood. In: Proc mechanized silvic workshop. Edmonton, Alberta, Canada, 29 February - 2 March 1984. For Can Northern For Res Cent Inf Rep NOR-X-272, 3739

Palmgren K (1984) Microbiological changes in forest soil following soil preparation and liming. Folia For (Helsinki) 603

Place ICM (1955) The influence of seed-bed conditions on the regeneration of spruce and balsam fir. Can Dep North Aff Nat Res For Div Bull 117

Plamondon AP, Ouellet DC, Déry G (1980) Effets de la scarification du site sur le microenvironnement. J Can Rech For 10, 476-482

Prévost M, Plamondon AP (1987) Interception de la pluie par les débris et la couverture végétale établie après la coupe à blanc. Nat Can 114, 127-132

Prévost M, Bolghari HA (1990) Croissance et enracinement de deux provenances d'épinette noire en fonction de la densité apparente du sol et de ses propriétés hydriques. $J$ Can Rech For 20, 185-192

Prévost M, Barry R, Stein J, Plamondon AP (1989) Évolution du couvert de neige et des profils hydrique et thermique du sol dans un bassin de la sapinière laurentidienne. $J \mathrm{Hy}$ drol 107, 343-366

Pritchett WL, Fisher RF (1987) Physical properties of forest soils. In: Properties and management of forest soils. J Wiley and Sons, New York, USA, 111-124

Raney WA, Edminster TW, Allaway WH (1955) Current status of research in soil compaction. Soil Sci Soc Am Proc 19, 423-428

Rawinski JJ, Bowles JA, Noste NV (1980) Soil properties related to coniferous seedling height growth in northern Wisconsin. USDA For Serv Res Note NC-254

Roberts HA (1981) Seed banks in soil. Adv Appl Biol 6, 1-55 
Robinson FC (1987) Alternate strip clearcutting in upland black spruce. 1 . An introduction. For Chron 63, 435-438

Robitaille L (1978) Résultats quinquennaux de plantation et de régénération naturelle du bouleau jaune, suite à une coupe rase avec et sans scarification. In: Proc Symp IUFRO. Nancy, France

Ross DW, Scott W, Heninger RL, Walstad JD (1986) Effects of site preparation on Ponderosa pine (Pinus ponderosa), associated vegetation, and soil properties in south central Oregon. J Can Rech For 16, 612-618

Ruel JC (1989) Importance de la régénération préexistante dans les forêts publiques du Québec. Ann Sci For 46, 345-359

Rutter AJ (1975) The hydrological cycle in vegetation. In: Vegetation and the atmosphere (JL Monteith, ed) Academic Press, New York, USA, 111-154

Salonius PO (1983) Effects of organic - mineral soil mixtures and increasing temperature on the respiration of coniferous raws humus material. J Can Rech For 13, 102-107

Sellers WD (1969) Physical climatology. Univ Chicago Press, Chicago, Illinois, USA

Shear CB, Crane HL, Myers AT (1946) Nutrientelement balance: a fundamental concept in plant nutrition. Proc Am Soc Hortic Sci 47, 239-248

Shultz RP (1975) Intensive culture of southern pines: maximum yield on short rotation. lowa State J Res 49, 325-337

Simmons GL, Pope PE (1987) Influence of soil compaction and vesicular - mycorr izae on root growth of yellow poplar and sweet gum seedlings. J Can Rech For 17, 970-975

Sims HP (1975) Temperature and moisture conditions on plowed Jack pine strip cut in southeastern Manitoba. J Can Rech For 5 , 541-545

Slay JM, Lockaby BG, Adams JC, Vidrine CG (1987) Effects of site preparation on soil physical properties, growth of Loblolly pine, and competing vegetation. South J Appl For 11, 83-86

Smith CR (1984) Status of mechanization of sitviculture in Canada. In: Proc symp mechanization silvic: increasing quality and productivity. Thunder Bay, Ontario, Canada, 17 September 1984. Can Pulp Pap Assoc 41-49
Smith GKM (1988) Site preparation in cold soils. Can For ind, September, 32-34

Stafford CW, Torbert JL, Burger JA (1985) An evaluation of site preparation methods for loblolly pine regeneration on the Piedmont. In: Proc 3rd biennial south silvic res conf. Atlanta, Georgia, USA, 7-8 November 1984. USDA For Serv Gen Tech Rep SO-54, 57-60

Stransky JJ, Roese JH, Watterson KG (1985) Soil properties and pine growth affected by site preparation after clearcutting. South $J$ Appl For 9, 40-43

Sutherland B (1987) La nécessité d'uniformatiser l'évaluation de l'équipement sylvicole. In: Proc $68^{e}$ Congrès Annu ACPPP. Montréal, Québec, Canada, 17-18 mars 1987. Can Pulp Pap Assoc 143-150

Sutton RF (1984) Mounding site preparation: evaluation of Jack pine outplantings in a boreal Ontario study. In: Proc Jack pine symp. Timmins, Ontario, Canada, 18-20 October 1983. For Can Great Lakes For Res Cent Symp Proc 0-P-12, 66-67

Sutton RF (1987) Root growth capacity and field performance of Jack pine and black spruce in boreal stand establishment in Ontario. $J$ Can Rech For 17, 794-804

Swindel BF, Conde LF, Smith JE (1984) Species diversity: concept measurement and response to clearcutting and site preparation. For Ecol Manage 8, 11-22

Tryon PR, Chapin FS III (1983) Temperature control over root growth and root biomass in taiga forest trees. J Can Rech For 13, 827833

Tuttle CL, Golden MS, Meldahl RS (1985a) Surface soil removal and herbicide treatment: effects on soil properties and Loblolly pine early growth. Soil Sci Soc Am J 49, 15581562

Tuttle CL, Golden MS, Meldahl RS (1985b) Site preparation effects on selected soil properties and early Loblolly pine seedling growth. In: Proc of the 3rd biennial southern silvic res conf. Atlanta, Georgia, USA, 7-8 November 1984. USDA For Serv Gen Tech Rep SO-54, 45-52

Van Cleve K, Barney R, Schlentner R (1981) Evidence of temperature control of production and nutrient cycling in two interior Alaska black spruce ecosystems. I Can Rech For $11,258-273$ 
Vitousek PM, Matson PA (1985) Intensive harvesting and site preparation decrease soil $\mathrm{ni}$ trogen availability in young plantations. South J Appl For 9, 120-125

Vitousek PM, Gosz JR, Grier CC, Melillo JM, Reiners WA, Tood RL (1979) Nitrate losses from disturbed ecosystems. Science 204, 469-474

Vomocil JA, Flocker WJ (1961) Effect of soil compaction on storage and movement of soil air and water. Trans Am Soc Agric Eng 4, 242-246

Voorhees WB, Allmara RR, Johnson CE (1981) Alleviating temperature stress. In: Modifying the root environment to reduce crop stress (GF Arkin, HM Taylor, eds), Am Soc Agric Eng, St-Joseph, Michigan, USA, 217-266

Waring RH, Schlesinger WH (1985) Decomposition and forest soil development. In: Forest ecosystems. Concepts and management. Academic Press, Orlando, Florida, USA, $181-210$
Wasterlund I (1985) Compaction of till soils and growth tests with Norway spruce and Scots pine. For Ecol Manage 11, 171-189

Wilhite LP, McKee WH (1985) Site preparation and phosphorus application alter early growth of Loblolly pine. South J Appl For 9, 103-109

Wittwer RF, Dougherty PM, Cosby D (1986) Effects of ripping and herbicide site preparation treatments on Loblolly pine seedling growth and survival. South J Appl For 10, 253-257

Wright JC (1985) Mechanization of silviculture on the St Regis (Alberta) LTD forest management area. In: Proc mechanized silvic workshop. Edmonton, Alberta, Canada, 29 February - 2 March 1984. For Can Northern For Res Cent Inf Rep NOR-X-272, 33-36

Zisa RP, Halverson HG, Stout BB (1980) Establishment and early growth of conifers on compact soils in urban area. USDA For Serv Res Pap NE-451 